\title{
Die Modellierungskonzepte von Wirtschaftssystemen
}

\author{
Zenon Glodek
}

Es werden im Folgenden einige Modellierungskonzepte erläutert, die man gemeinsam als systemdynamische Modellbildung bezeichnet. $\mathrm{Zu}$ diesem Bereich gehören folgende Modellierungsansätze: System-Dynamics-Verfahren, FLOR-Modellierung, Regelkreisoptimierungsmodelle und Dynamisches Fortschreibungsmodell. Wann immer man versucht, ein Wirtschaftssystem zu analysieren und in Differenzial- bzw. Differenzengleichungen abzubilden, ist systemdynamische Modellierung ein gangbarer Weg. Sie basiert explizit auf der soft-modeling Simulationsmethode, die die wirtschaftlichen Reproduktionsprozesse im globalen Rahmen abzubilden und in ihrer dynamischen Interaktion zu interpretieren erfordert.

\section{System Dynamics}

System Dynamics ist eine Modellierungskonzeption dynamischer Systeme, die von Jay W. Forrester am MIT entwickelt wurde. Sie geht von einer bestimmten Interpretationsweise aus. Hiernach können wirtschaftliche Reproduktionsprozesse stets durch bestimmte zeitveränderliche Bestandgrößen (Level) und deren verzögert beeinflusste $\mathrm{Zu}$ - und Abgänge (Rates) beschrieben werden. Dieser Interpretationsweise ist die Programmiersprache DYNAMO angepasst, die die sprachlichen Kategorien zu einer einfachen und computergestuitzten Formulierung von systemdynamischen Modellen liefert.

Zur Darstellung einer im SD-Konzept interpretierten Welt verwendet man besondere Diagrammsymbole und Darstellungskonvektionen. Anhand dieser Darstellungskonvektionen formuliert man bestimmte Gleichungstypen, die die Berechnung des Modells auf dem Computer ermöglichen.

Bei der Modellierung verwendet man bestimmte Parameter, die die Verzögerung, die informationelle Verknuipfung, die exponentielle Glättung sowie Störungen zum Ausdruck bringen. Die Beschreibung der Raten und Hilfsgleichungen erfolgt in Form der empirischen Hypothesen, die durch bestimmte Tabellenfunktionen formuliert sind.

Ein weiteres konzeptionelles Element der DS-Modellierung bezieht sich auf die Art der Hypothesengewinnung. Wir haben erwähnt, dass sich alle Reproduktionsprozesse als ein Geflecht von Feed-back-Kreisen deuten lassen. Diese Vorgehensweise zur Hypothesengewinnung bezeichnet man als Feed-back-Heuristik. Diese Forde- rung zur Feststellung der Feed-back-Kreise kann so verstanden werden, dass nicht alle in dem System wirkenden Feed-backs zu ermitteln seien, sondern nur die als wesentlich erachteten. Aus dieser Feststellung ergibt sich sofort die Frage nach Systemgrenzen. Man wird gefragt: Wo ist die Grenze, die die kleinste Anzahl von Elementen umschließt, innerhalb derer das zu untersuchende dynamische Verhalten des Systems erzeugt wird [1]. Eine Systemabgrenzung wird in der Form eines komparativen Kausaldiagrammes abgebildet. Das Modell bildet eine Struktur von zusammenwirkenden Rückkopplungsschleifen ab, die innerhalb der geschlossenen Systemgrenzen die bestehenden Elemente darstellen. Dabei haben wir es mit einer Maxime zu tun, die als Geschlossenheitsprinzip bezeichnet wird. Nach diesem Prinzip darf in einem Modell nur eine zeitveränderliche exogene Variable existieren, die durch eine Störungsfunktion abgebildet wird. Durch die vorgeplante Veränderung der Störungsgröße erfolgt eine bestimmte Testantwortanalyse. Man versucht, nach der Formulierung einer Störung das Modellverhalten im Hinblick auf bestimmte wünschenswerte Eigenschaften zu verändern. Wenn die Parameteränderungen im Modell zu dem gewünschten Erfolg geführt haben, dann werden sie am konkreten System realisiert. Oft wird dabei eine sogenannte Szenariotechnik angewandt.Das Szenario ist die exogene Vorgabe langfristiger Änderungen bestimmter Variablen oder Parameter.

Die Berechnung der Bestandsgrößen erfolgt durch das Multiplizieren aller Zu- und Abgänge mit DT-Zeitinkrement. In einem die Realität möglichst weitgehend beschreibenden SD-Modell muß DT $\rightarrow 0$ streben, d. h. infinitesimal klein sein. Diese Infinitesimalprämisse wird stark kritisiert [2]. Es wird behauptet, dass in den meisten Fällen einer Modellentwicklung vielmehr eine angemessene Realitätsbeschreibung erst durch die Annahme eines diskreten äquidistanten Zeitsystems möglich werden dürfte.

\section{FLOR-Modellierung}

Auf der Basis der Kritik von SD-Verfahren ist eine alternative Konzeption entwickelt worden. Diese schrittweise entwickelte Konzeption wird als FLOR-Modellierung/ feed-back-orientierte offene Level-Raten-Modellierung bezeichnet [3]. In der ersten Stufe wird die in dem SDKonzept immanente Infinitesimalprämisse durch eine Diskretzeitprämisse ersetzt. Die zweite Stufe führt zur Verwerfung des im SD-Konzept enthaltenen Prinzips, dass informationelle Beziehungen von Informations- 
leveln stammen. Im Rahmen der dritten Stufe wird das von der SD-Konzeption geforderte Geschlossenheitsprinzip durch ein umfassendes Offenheitsprinzip abgelöst. Die FLOR-Modellierung gestattet wegen der eindeutigen Periodenzuordnung des Zeitindizes die Verwendung sämtlicher einschlägiger Parameterschätztechniken, die für rekursive Differenzengleichungsmodelle im Rahmen der Ökonometrie entwickelt wurden.

\section{Regelkreisoptimierungsmodell}

Für die Effektivität der wirtschaftlichen Reproduktionsprozesse soll die heuristische Optimierung von SD-Modellen von Bedeutung werden. Ein Regelkreisoptimierungsmodell/ROM basiert auf der Steuerung des Optimierungsverfahrens und dem Verhalten des SD-Modells anhand der expliziten Vorgabe einer Zielfunktion [4].

Das SD-Modell wird als Regelstrecke in die Regelkreisstruktur integriert. Der Steuervektor s/t/ bildet die Eingangsgröße des SD-Modells. Die Elemente $s_{i} / t /$ des Steuervektors werden durch untere und obere Restriktionen begrenzt. Der Steuervektor $\underline{s} / \mathrm{t} /$ wird durch den Optimierungsalgorithmus über den Simulationszeitraum $\mathrm{t}_{\mathrm{o}} \leq \mathrm{t} \leq \mathrm{t}_{\max }$ in der Weise bestimmt, dass die Differenz zwischen den Komponenten des Ausgangsvektors z/s, t/ $=z_{1} / \underline{s}, t /, z_{2} / \underline{s}, t /,, z_{n} / s, t /$ und den entsprechender Komponenten der Zielfunktionen $U(\underline{s}, t)$ für $t_{0} \leq t \leq t_{\max }$ innerhalb vorgegebener Schranken liegt.

Die Anwendbarkeit von bestimmten Modellierungskonzepten hängt von der verfügbaren Software ab. Die Softwareentwicklung uibt einen großen Einfluss auf die Handhabung und Validierung der systemdynamischen Modelle aus. Eine Entwicklungstendenz erfolgt in Form des allgemeinen Modelltyps „Dynamisches Fortschreibungsmodell“, der in einem Modellbanksystem unterstiitzt wird.

\section{Dynamische Fortschreibungsmodelle}

Dynamische Fortschreibungsmodelle können durch die schrittweise Berechnung einer Datenmatrix gekennzeichnet werden. In den Spalten dieser Matrix sind die Variablen (auch mehrdimensionale Matrizen) angeordnet, die Ziele entsprechen aufeinenderfolgenden, äquidistanten Zeitpunkten. Für die „Input-Variablen“ sind alle Werte für den zu untersuchenden Zeitraum schon vor der Simulation gegeben. Für die „Output-Variablen“ werden die Werte während der Simulation berechnet und sind teilweise nur für die ersten Zeitpunkte (Lagwerte) vorhanden. Diese Berechnung erfolgt schrittweise durch einen sogenannten Modelloperator. In Abhängigkeit von Zwischenergebnissen kann die Simulationszeit auch zurückgesetzt werden. MBS bietet die Möglichkeit, einige Zeitreihen von statistischen Daten einer zentralen Methodenbank mathematisch-statistischer Verfahren und eine Modellbank von verschiedenen Modelltypen bei der Problemlösung parallel zu nutzen.

\section{Anmerkungen}

[1] J. W. Forrester, Principles of Systems, MIT Press, Cambridge

[2] E. Zwicker, Simulation und Analyse dynamischer Systeme in den Wirtschafts- und Sozialwissenschaften, WdeG, Berlin West/New York 1981, S. 488

[3] E. Zwicker, op. cit. S.522

[4] H. Krallmann, Der Modell-Methodenverband in der Systemtheorie, in: Systemtheorie in Wirtschaft und Verwaltung, J. Baet, et. al., Duncker \& Humblot, Berlin West 1977, S. 288

\author{
Autor \\ prof. dr hab. Zenon Glodek \\ Panstwowa Wyzsza Szkola Zawodowa w Gorzowie Wlkp. \\ ul. Teatralna 25 \\ 66-400 Gorzów Wlkp., Polen \\ Tel. +48 (95) 721 60-32 \\ Fax +48 (95) $72160-22$
}

